Manoel Afonso Guimarães Gonçalves'

FERNANDO ANSCHAU ${ }^{2}$

ChrYSTIANE MARC ${ }^{3}$

LUISE MEURER ${ }^{4}$

\title{
Adenocarcinoma viloglandular de cérvice uterina
}

\author{
Villoglandular adenocarcinoma of the uterine cervix
}

\section{Palavras-chaves \\ Adenocarcinoma Neoplasias do colo do útero \\ Prognóstico}

Keywords

Adenocarcinoma Uterine cervical neoplasms/therapy

Prognosis
Correspondência:

Manoel Afonso Guimarães Gonçalves Centro Clínico da Pontifícia Universidade Católica do Rio Grande do SUl - PUC-RS Avenida Ipiranga, 6.690 - cj. 217 CEP 90610000 - Porto Alegre/RS Fone/Fax: (51) 3339-2222 E-mail: mafonsog@terra.com.br

Recebido 03/09/2007

\section{Resumo}

OBJETIVO: o adenocarcinoma viloglandular (AVG) da cérvice foi identificado como uma variante do adenocarcinoma cervical que ocorre em mulheres jovens e traz um excelente prognóstico. Diante da escassez de estudos relacionados ao tema, nós relatamos seis casos de AVG de cérvice. MÉTODOS: acompanhamos a evolução de seis casos de AVG, no período de 1995 a 2006, no Hospital São Lucas da Pontifícia Universidade Católica do Rio Grande do Sul (PUC-RS). Coletamos informações clínicas e histológicas de todas as pacientes e submetemos todas as peças cirúrgicas para revisão histológica. RESULTADOS: a idade média da apresentação foi de 43,5 anos (variando de 27 a 61 anos). Quatro pacientes submeteram-se à histerectomia radical de Wertheim-Meigs e linfadenectomia pélvica bilateral, uma submeteu-se a conização e subseqüente radioterapia e uma a linfadenectomia pélvica seguida de radioterapia. Todas as pacientes estão vivas e bem, sem evidência de recorrência. CONCLUSÕES: as implicações da terapia são discutidas. Propomos aqui a inclusão do estudo do padrão de envolvimento linfovascular na determinação diagnóstica do AVG. Assim, ao referenciarmos este diagnóstico, poderemos optar, com cautela, pela terapia conservadora, salvo particularidades de cada caso.

\section{Abstract}

PURPOSE: the villoglandular adenocarcinoma (VGA) of the cervix has been identified as a variant of cervical adenocarcinoma that occurs in young women, which has an excellent prognosis. Considering the scarcity of studies related to the subject, we report six cases of VGA of the cervix. METHODS: we followed the development of six cases of VGA in the period from 1995 to 2006 at Hospital São Lucas of Pontifícia Universidade Católica do Rio Grande do Sul (PUC-RS). We collected clinical and histologic information of the patients and submitted all the surgical specimens to histological review. RESULTS: mean age at diagnosis was 43.5 years (range 27-61 years). Four patients were submitted to Wertheim-Meigs radical hysterectomy and bilateral pelvic lymphadenectomy, one to conization and subsequent radiotherapy and one to pelvic lymphadenectomy followed by radiotherapy. All the patients were alive and well at the time of this writing, without evidence of recurrence. CONCLUSIONS: the implications of therapy are discussed. We propose here the inclusion of the study of the pattern of lymphovascular involvement in determining the diagnosis of VGA. Thus, in referring to this diagnosis, we will be able to opt, with caution, for conservative therapy, except for particularities of each case.

\section{Serviço de Ginecologia do Hospital São Lucas da Pontifícia Universidade Católica do Rio Grande do Sul - PUC-RS - Rio Grande} do Sul (RS), Brasil.

' Professor Adjunto da Faculdade de Medicina da Pontifícia Universidade Católica do Rio Grande do Sul - PUC-RS - Rio Grande do Sul (RS), Brasil; Responsável pelo Setor de Oncologia Pélvica do Serviço de Ginecologia do Hospital São Lucas da Pontifícia Universidade Católica do Rio Grande do Sul - PUC-RS - Rio Grande do Sul (RS), Brasil.

${ }_{2}^{2}$ Médico Assistente Membro do Serviço de Ginecologia do Hospital São Lucas da Pontifícia Universidade Católica do Rio Grande do Sul PUC-RS - Rio Grande do Sul (RS), Brasil. Coordenador do Serviço Integrado de Ginecologia de Alvorada e Cachoeirinha (SIGAC). Professor das Faculdades de Enfermagem, Nutrição e Fonoaudiologia Nossa Senhora de Fátima de Caxias do Sul - Caxias do Sul (RS), Brasil.

${ }_{3}^{3}$ Médica Assistente Membro do Serviço de Ginecologia do Hospital São Lucas da Pontifícia Universidade Católica do Rio Grande do

Sul - PUC-RS - Rio Grande do Sul (RS), Brasil. Membro do Serviço Integrado de Ginecologia de Alvorada e Cachoeirinha (Sigac).

${ }^{4}$ Professora Adjunto do Departamento de Patologia da Universidade Federal do Rio Grande do Sul - UFRGS - Rio Grande de Sul (RS), Brasil. 


\section{Introdução}

Estudos epidemiológicos têm demonstrado um aumento na incidência de adenocarcinomas de cérvice uterina, especialmente entre mulheres com até 35 anos de idade ${ }^{1-4}$. Um estudo norueguês demonstrou o aumento do adenocarcinoma cervical de $38 \%$ entre 1970 e 1984, com elevação nas taxas restritas às mulheres entre 20 e 34 anos de idade 5 .

Segundo dados do Instituto Nacional do Câncer do Brasil (Inca), o adenocarcinoma corresponde à segunda neoplasia mais freqüente do colo uterino, com prevalência de 12 a 15\% $\%^{6}$ Um estudo na cidade de Porto Alegre avaliou 1.137 casos de câncer cervical no período de 1995 a 2003 , encontrando $20,1 \%$ de adenocarcinomas ${ }^{4}$.

$\mathrm{O}$ adenocarcinoma de cérvice uterina apresenta pior prognóstico em relação ao carcinoma epidermóide. Os tumores glandulares do colo uterino podem ser classificados em seis tipos histológicos: adenocarcinoma sem outras especificações, adenocarcinoma mucinoso, endometrióide, de células claras, seroso e mesonéfrico. $\mathrm{O}$ carcinoma mucinoso responde por $70 \%$ dos casos e é subdividido em cinco subtipos: endocervical, intestinal, de células em anel de sinete, de desvio mínimo e viloglandular ${ }^{7,8}$.

$\mathrm{O}$ adenocarcinoma viloglandular (AVG) foi descrito pela primeira vez por Young e Scully ${ }^{9}$, em 1989, como uma variante relativamente rara de adenocarcinoma endocervical bem diferenciado. Afeta usualmente mulheres no menacme com idade entre 33 e 37 anos. Entretanto, outros autores encontraram pacientes com este diagnóstico e idade média de $45 \operatorname{anos}^{10,11}$. A incidência tem sido estimada entre 2 e $5 \%$ dos adenocarcinomas de cérvice ${ }^{9,12,13}$. O prognóstico do AVG foi relatado como melhor em relação a outros tipos de adenocarcinoma ${ }^{9,12,13}$.

O reconhecimento de células de AVG na citologia de papanicolaou é bastante difícil, pois traz algumas similaridades citomorfológicas tanto com células colunares reativas quanto com o adenocarcinoma in situ. Além disto, por vezes apresenta apenas atipias glandulares leves ${ }^{14,15}$.

Tendo em vista a raridade da doença em questão, o presente estudo traz uma análise clínico-patológica de seis casos de pacientes com diagnóstico inicial de AVG e a revisão da literatura pertinente.

\section{Métodos}

Realizamos um estudo retrospectivo, no qual avaliamos a evolução de seis casos diagnosticados inicialmente como AVG no período de 1995 a 2006 no Hospital São Lucas da Pontifícia Universidade Católica do Rio Grande do Sul (PUC-RS). Foram incluídas no estudo todas as pacientes cujo diagnóstico histológico inicial, firmado por biópsia ou conização de colo uterino, foi de AVG. Coletamos informações clínicas e histológicas de todas as pacientes e submetemos todas as peças cirúrgicas à revisão histológica com um segundo médico patologista.

\section{Resultados}

A média de idade das pacientes foi de 43,5 anos (27 a 61 anos). Os sinais e sintomas que levaram ao diagnóstico foram variados. Duas pacientes procuraram atendimento por alteração no exame citopatológico cervical, uma apresentou sangramento na pós-menopausa, uma foi encaminhada por lesão polipóide no colo uterino e outras duas apresentavam sinusorragia. Em nosso exame ginecológico, observamos colo uterino ulcerado em quatro pacientes, alteração colposcópica sugestiva de lesão intraepitelial cervical de alto grau (HSIL) em uma paciente e lesão polipóide cervical em outra. Apenas duas pacientes faziam uso regular de anticoncepcionais orais, mas nenhuma paciente encontravase grávida no momento do diagnóstico. O número médio de gestações e partos foi três; contudo, a paciente mais jovem (27 anos) era nuligesta (Tabela 1).

Tabela 1 - Pacientes com diagnóstico inicial de adenocarcinoma viloglandular.

\begin{tabular}{|c|c|c|c|c|c|c|c|c|c|c|}
\hline Caso & $\begin{array}{l}\text { Idade } \\
\text { (anos) }\end{array}$ & Paridade & $\begin{array}{l}\text { Sinais e } \\
\text { sintomas }\end{array}$ & $\begin{array}{l}\text { Diagnóstico inicial } \\
\text { de AVG obtido por }\end{array}$ & Estádio & Tratamento & $\begin{array}{c}\text { AP } \\
\text { Linfonodos }\end{array}$ & $\begin{array}{l}\text { Envolvimento do } \\
\text { espaço linfovascular }\end{array}$ & $\begin{array}{c}\text { AP } \\
\text { definitivo }\end{array}$ & $\begin{array}{c}\text { Seguimento } \\
\text { (meses) }\end{array}$ \\
\hline 1 & 27 & GOPO & Alteração citológica & Conização & $\mathrm{IB}$ & WM & 14 negativos & Não & AVG & 120 \\
\hline 2 & 61 & G8P7A1 & $\begin{array}{l}\text { Sangramento } \\
\text { pós-menop }\end{array}$ & Biópsia & $\mathbb{I B}$ & WM & 18 negativos & Não & AVG & 72 \\
\hline 3 & 54 & G2P2 & Sinusorragia & Biópsia & $\| A$ & Conização + Rxt + Braqui & - & Não & AVG & 36 \\
\hline 4 & 49 & G4P4 & Lesão polipóide & $\begin{array}{l}\text { Polipectomia + } \\
\text { conização }\end{array}$ & $\mathbb{I B}$ & WM & 20 negativos & Não & AVG & 12 \\
\hline 5 & 35 & G4P4 & Sinusorragia & Biópsia & $\mathbb{I B}$ & Linf + Rxt + Braqui & $\begin{array}{c}2 \text { positivos em } 14 \\
\text { examinados }\end{array}$ & Não & AVG & 24 \\
\hline 6 & 35 & G1P1 & Alteração citológica & Biópsia & $\mathbb{B}$ & WM & 47 negativos & Sim & $\begin{array}{l}\text { Adenocarcinoma } \\
\text { mucinoso de tipo } \\
\text { endocervical }\end{array}$ & 12 \\
\hline
\end{tabular}

$\mathrm{G}=$ gestações; $P=$ partos; $A=a b o r t o s ; ~ P o ́ s-m e n o p=$ pós-menopáusico; $A P=e x a m e$ anátomo-patológico; $W M=$ Wertheim Meigs; Lnf=Linfadenectomia; Rxt=Radioterapia;

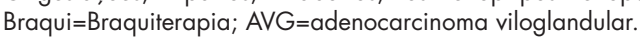


O diagnóstico inicial de AVG foi firmado por meio de biópsia do colo uterino em quatro casos (Casos 2, 3, 5 e 6). Em um caso (Caso 1), a biópsia cervical demonstrou apenas lesão intraepitelial de alto grau e o diagnóstico inicial de AVG ocorreu com a conização. Em outro caso, com apresentação clínica de lesão polipóide, o diagnóstico foi firmado com conização após a polipectomia ter sugerido AVG (Tabela 1).

Após avaliação clínica, o estádio de cinco pacientes era IB e uma foi classificada como IIA. As pacientes submetidas inicialmente à conização (uma com biópsia cervical sugestiva de HSIL e outra com lesão polipóide cervical) posteriormente foram tratadas com cirurgia de Wertheim-Meigs. Outra paciente (Caso 2), com diagnóstico por biópsia cervical de AVG, foi submetida à cirurgia de Wertheim-Meigs. Uma paciente (Caso 5), com biópsia cervical evidenciando AVG e cujo planejamento cirúrgico era de WertheimMeigs, submeteu-se apenas a linfadenectomia pélvica, cuja avaliação trans-operatória demonstrou comprometimento tumoral em dois linfonodos de 14 examinados, razão pela qual foi encaminhada para radioterapia e braquiterapia.

Outra paciente, com sinusorragia e com biópsia cervical sugerindo AVG, foi submetida à conização, cujo diagnóstico histológico definitivo confirmou AVG com margens cirúrgicas comprometidas. Esta paciente foi classificada como em estádio clínico IIA e encaminhada para tratamento complementar com radioterapia e braquiterapia.

O espaço linfovascular estava comprometido em uma paciente (Caso 6), cujo diagnóstico definitivo demonstrou tratar-se de adenocarcinoma invasor mucinoso de tipo endocervical e não AVG (diagnóstico inicial de AVG por biópsia cervical e tratamento definitivo com cirurgia de Wertheim-Meigs). As outras cinco pacientes não apresentavam comprometimento do espaço linfovascular e o diagnóstico definitivo confirmou AVG bem diferenciado.

A linfadenectomia pélvica das quatro pacientes submetidas à cirurgia de Wertheim-Meigs não evidenciou qualquer metástase tumoral em 99 linfonodos examinados, média de 24,7 linfonodos por paciente. Todas as peças cirúrgicas obtidas da cirurgia de Wertheim-Meigs apresentaram margens cirúrgicas livres de neoplasia.

O seguimento médio das pacientes foi de três anos e oito meses, sendo que uma das pacientes já completara o seu tratamento há 120 meses. Todas as pacientes mantiveram acompanhamento sem evidência de recidiva tumoral até a data de produção deste artigo (Tabela 1).

\section{Discussão}

O AVG é uma forma bem diferenciada de adenocarcinoma cervical que ocorre predominantemente em mulheres jovens ${ }^{16}$. Neste adenocarcinoma, podemos observar três quadros morfológicos principais: proliferação exofítica, arquitetura papilar e leve a moderada atipia $^{9,12,17,18}$. Apresenta um padrão arborescente, com papilas de eixo central fibroso, revestidas de células colunares estratificadas ${ }^{9,13,19,20}$. Após a primeira descrição da doença em $1989^{9}$, mais de 100 casos já foram relatados na literatura.

A maioria destes relatos, independente dos achados, teve um bom prognóstico. Este prognóstico favorável, juntamente com a idade das pacientes na qual o tumor é diagnosticado, traz a possibilidade de que pacientes com desejo de preservar a fertilidade possam ser manejadas com tratamento conservador por meio da conização do colo uterino ${ }^{9,12}$.

A média de idade das nossas pacientes foi de 43,5 anos, correspondendo à média etária encontrada por alguns autores ${ }^{9,12}$. Apenas três pacientes se encontravam em idade reprodutiva - uma das quais era nuligesta. É interessante observar a ampla faixa etária, de 27 a 61 anos, assim como a heterogeneidade da sintomatologia apresentada nas nossas pacientes. Esta ausência de padrão na apresentação clínica traduz a dificuldade na determinação diagnóstica da patologia. O quadro clínico heterogêneo faz com que os tratamentos propostos sejam diversos, variando do tratamento conservador, radioterapia, quimioterapia, até o tratamento radical cirúrgico ${ }^{9,12,13,16,21}$. Nenhuma das pacientes deste estudo foi manejada com tratamento conservador e todas apresentaram excelente prognóstico pós-operatório (Tabela 1). Entretanto, ainda é incerto se o prognóstico favorável deve-se ao fato de os tumores serem bem diferenciados e/ou por se apresentarem em estágios iniciais.

Ao contrário da maioria dos estudos, o prognóstico relatado por outros autores não foi bom. Um caso com múltiplas recorrências cinco anos após o tratamento cirúrgico inicial, dois casos com metástases em um estudo com sete pacientes e outros dois casos de metástases em 15 pacientes, além de outro com recorrência tumoral na cicatriz de episiorrafia, quatro anos após parto normal e histerectomia radical com linfadenectomia pélvica e para-aórtica fazem parte destes relatos com prognósticos ruins ${ }^{22-25}$. Esta série de casos com prognósticos desfavoráveis relacionados na literatura chama atenção para o fato de a patologia desta doença ainda ser obscura.

A maioria dos relatos de metástases e mau prognóstico fogem de uma conceituação mais detalhista para o AVG. 
Nós propomos exatamente este tipo de conceituação, com o diagnóstico detalhado da entidade nosológica por meio da histologia revisada, por um segundo médico patologista, em peça de conização com margens cirúrgicas livres de neoplasia e sem envolvimento do espaço linfovascular. Os relatos com prognósticos reservados recaem sobre aqueles casos com envolvimento do espaço linfovascular, o não conhecimento da doença na peça de conização e até mesmo na associação com outras neoplasias. Estes relatos sairiam, desta maneira, de um conceito pleno de AVG.

No nosso grupo, encontramos envolvimento do espaço linfovascular em apenas uma paciente, cujo diagnóstico de AVG não foi confirmado na revisão histológica. Este caso retrata precisamente a importância da revisão adequada da peça cirúrgica. Aqui, o caso seria excluído do grupo com AVG pelo envolvimento do espaço linfovascular e pela revisão diagnóstica, que demonstrou tratar-se de um adenocarcinoma mucinoso de tipo endocervical.

Acreditamos que a ausência de comprometimento linfovascular, quando do exame de todo o colo uterino, seja um fator determinante e diagnóstico nos casos de AVG. Esta doença, com raras exceções na literatura, comporta-se de maneira favorável e sem metástases, em especial quando não há envolvimento do espaço linfovascular. Portanto, propomos a inclusão do estudo do padrão de envolvimento linfovascular na determinação diagnóstica do AVG. Desta forma, ao referenciarmos este diagnóstico, poderemos optar, com cautela, pela terapia conservadora, salvo particularidades de cada caso.

Assim como já enfatizamos, outros autores também concordam que o diagnóstico do AVG de colo uterino só poderia ser realizado em peças de conização cervical ou espécimes de histerectomia ${ }^{3}$. A paciente com envolvimento linfonodal não foi submetida à conização, o que prejudica a análise de seu caso (Tabela 1). Esta paciente seria excluída da conceituação de AVG, por não apresentar a revisão histológica em uma peça de conização cervical, além de apresentar comprometimento linfovascular. Uma biópsia cervical pode indicar que o crescimento é do tipo viloglandular, mas a conização é mandatória quando não podemos identificar se o tumor é um AVG puro ${ }^{10}$. Mesmo que áreas focais de aspecto viloso estejam presentes em outros tipos de adenocarcinoma cervical, este diagnóstico é reservado apenas aos casos com componente histológico quase que inteiramente viloglandular.

Alguns autores têm enfatizado a importância de distinguir o adenocarcinoma endocervical com componente viloglandular do AVG bem diferenciado. Isto deve ser feito com base nas diferenças da arquitetura papilar e do grau de atipia citológica. Tumores viloglandulares são caracteristicamente papilares e têm apenas leve a mínima atipia citológica, comparados aos endocervicais primários ${ }^{9,12,26-28}$. Devido à raridade deste subtipo histológico, a normatização do diagnóstico afeta o número de relatos de caso e a abordagem terapêutica ${ }^{26-28}$.

Existem alguns critérios que definem as circunstâncias clínico-patológicas sobre as quais o manejo conservador do AVG pode ser considerado: o tumor tem que ser superficial; as margens de excisão do tumor, negativas; ausência de envolvimento do espaço vascular e, a paciente deve aderir ao segmento ${ }^{9}$. A estes critérios deveríamos acrescentar a revisão histológica sistemática das peças de conização, nas quais o diagnóstico definitivo será baseado, e, assim, teremos a entidade nosológica AVG de colo uterino firmada.

Desta maneira, excluiríamos três pacientes de nosso estudo, os Casos 3, 5 e 6 (Tabela 1): um caso (Caso 3) com margens cirúrgicas da conização comprometidas, outro (Caso 5) sem conização para revisão de toda a peça cirúrgica e um (Caso 6) com diagnóstico definitivo não confirmando AVG e com envolvimento do espaço linfovascular. Com este rigor na determinação diagnóstica, extraímos a conceituação pretendida do AVG: paciente com diagnóstico histológico revisado de AVG puro (sem outra neoplasia maligna associada) em peça de conização com margens livres, sem envolvimento do espaço linfovascular e com bom seguimento clínico. Só desta forma poderemos ter maior convicção na indicação de um tratamento conservador para estas pacientes.

\section{Referências}

1. Schwartz SM, Weiss NS. Increased incidence of adenocarcinoma of the cervix in young women in the United States. Am J Epidemiol. $1986 ; 124(6): 1045-7$

2. Anton-Culver $H$, Bloss JD, Bringman D, Lee-Feldestein A, DiSaia $P$, Manetta A. Comparison of adenocarcinoma and squamous cell carcinoma of the uterine cervix: a population-based epidemiologic study. Am J Obstet Gynecol. 1992;166(5):1507-14.
3. Alfsen GC, Thoresen SO, Kristensen GB, Skovlund E, Abeler VM. Histopathologic subtyping of cervical adenocarcinoma reveals increasing incidence rates of endometrioid tumors in all age groups: a population based study with review of all nonsquamous cervical carcinomas in Norway from 1966 to 1970,1976 to 1980 , and 1986 to 1990 . Cancer. 2000;89(6): $1291-9$. 
4. Cambruzzi E, Zettler CG, Pereira CAO. Adenocarcinoma endocervical em Porto Alegre e região metropolitana: morfologia e prevalência. Rev AMRIGS. 2005;49(1):27-33.

5. Eide TJ. Cancer of the uterine cervix in Norway by histologic type, 1970-84. J Natl Cancer Inst. 1987;79(2): 199-205.

6. Instituto Nacional de Câncer [homepage da Internet]. Rio de Janeiro: INCA; 1996-2007 [citado 20 jan 2007]. Disponível em: http://www.inca.gov.br

7. Colgan TJ, Auger M, McLaughlin JR. Histopathologic classification of cervical carcinomas and recognition of mucin-secreting squamous carcinoma. Int J Gynecol Pathol. 1993;12(1):64-9.

8. Smith HO, Tiffany MF, Qualls CR, Key CR. The rising incidence of adenocarcinoma relative to squamous cell carcinoma of the uterine cervix in the United States-a 24-year population-based study. Gynecol Oncol. 2000;78(2):97-105.

9. Young RH, Scully RE. Villoglandular papillary adenocarcinoma of the uterine cervix. A clinicopathologic analysis of 13 cases. Cancer. 1989;63(9): 1773-9.

10. Utsugi K, Shimizu Y, Akiyama F, Umezawa S, Hasumi K. Clinicopathologic features of villoglandular papillary adenocarcinoma of the uterine cervix. Gynecol Oncol. 2004;92(1):64-70.

11. Yamazawa K, Matsui H, Seki K, Mitsuhashi A, Kawamata Y, Shirasawa $H$, et al. Human papillomavirus positive well-differentiated villoglandular adenocarcinoma: a case report and review of literature. Gynecol Oncol. 2000;77(3):473-7.

12. Jones MW, Silverberg SG, Kurman RJ. Well-differentiated villoglandular adenocarcinoma of the uterine cervix: a clinicopathologic study of 24 cases. Int J Gynecol Pathol. 1993; 12(1): 1-7.

13. Bouman A, Oosterhuis GJ, Naudin ten Cate L, van Doorn GA. Villoglandular papillary adenocarcinoma of the cervix. Beware of a wolf in sheep's clothing. Eur J Obstet Gynecol Reprod Biol. 1999;87(2): 183-9.

14. Chang WC, Matisic JP, Zhou C, Thomson T, Clement PB, Hayes MM. Cytological features of villoglandular adenocarcinoma of the uterine cervix: comparison with typical endocervical adenocarcinoma with villoglandular component and papillary serous carcinoma. Cancer. 1999;87(1):5-11.

15. Wiesenfeld $U$, Baraggino $E$, Melato $M$. Adenocarcinoma villoghiandolare del collo uterino. Descrizione di un caso e analisi della letteratura. Minerva Ginecol. 1997;49(5):221-4.

16. Al-Nafussi Al, Hughes DE. Histological features of CIN 3 and their value in predicting invasive microinvasive squamous carcinoma. J Clin Pathol. 1994;47(9):799-804.
17. Garcea A, Nunns D, Ireland D, Brown L. A case of villoglandular papillary adenocarcinoma of the cervix with lymph node metastasis. BJOG. 2003;1 10(6):627-9.

18. Collinet $\mathrm{P}$, Prolongeau JF, Vaneecloo S. Villoglandular papillary adenocarcinoma of the uterine cervix. Eur J Obstet Gynecol Reprod Biol. 1999;86(1):101-3.

19. Young RH, Clement PB. Endocervical adenocarcinoma and its variants: their morphology and differential diagnosis. Histopathology. 2002;41(3): 185-207.

20. Stanley-Christian $H$, Heim BK, Hines JF, Hall KL, Willett GD, Barnes WA. Villoglandular adenocarcinoma of the cervix: a report of three cases and review of the literature. Gynecol Oncol. 1997;66(2):327-30.

21. Nagai N, Hirata E, Kusuda T, Mukai K, Arihiro K, Ohama K. Villoglandular papillary adenocarcinoma of the uterine cervix responding to neoadjuvant chemotherapy with docetaxel and cisplatin: a case report. Int J Gynecol Cancer. 2005;15(6):1 187-90.

22. Dede M, Deveci G, Deveci MS, Yenen MC, Goktolga U, Dilek $S$, et al. Villoglandular papillary adenocarcinoma of the uterine cervix in a pregnant woman: a case report and review of literature. Tohoku J Exp Med. 2004;202(4):305-10.

23. Heron DE, Axtel A, Gerszten K, Amortegui A, Kelley J, Comerci $\mathrm{J}$, et al. Villoglandular adenocarcinoma of the cervix recurrent in an episiotomy scar: a case report in a 32 -year-old female. Int J Gynecol Cancer. 2005; 15(2):366-71

24. Kaku T, Kamura T, Shigematsu T, Sakai K, Nakanami N, Uehira K, et al. Adenocarcinoma of the uterine cervix with predominantly villoglandular papillary growth pattern. Gynecol Oncol. 1997;64(1):147-52.

25. Khunamornpong S, Maleemonkol S, Siriaunkgul S, Pantusart A. Well-differentiated villoglandular adenocarcinoma of the uterine cervix: a report of 15 cases including two with lymph node metastasis. J Med Assoc Thai. 2001;84(6):882-8.

26. Macdonald RD, Kirwan J, Hayat K, Herrington CS, Shawki H. Villoglandular adenocarcinoma of the cervix: clarity is needed on the histological definition for this difficult diagnosis. Gynecol Oncol. 2006;100(1):192-4.

27. Fadare $O$, Zheng W. Well-differentiated papillary villoglandular adenocarcinoma of the uterine cervix with a focal high-grade component: is there a need for reassessment? Virchows Arch. $2005 ; 447(5): 883-7$.

28. Heatley MK. Villoglandular adenocarcinoma of the uterine cervix - a systematic review of the literature. Hystopathology. 2007;5 1 (2):268-9 